\title{
Hybrid grating reflectors: Origin of ultrabroad stopband
}

\author{
Park, Gyeong Cheol; Taghizadeh, Alireza; Chung, II-Sug
}

Published in:

Applied Physics Letters

Link to article, DOI:

$10.1063 / 1.4945737$

Publication date:

2016

Document Version

Publisher's PDF, also known as Version of record

Link back to DTU Orbit

Citation (APA):

Park, G. C., Taghizadeh, A., \& Chung, I-S. (2016). Hybrid grating reflectors: Origin of ultrabroad stopband. Applied Physics Letters, 108(14), [141108 ]. https://doi.org/10.1063/1.4945737

\section{General rights}

Copyright and moral rights for the publications made accessible in the public portal are retained by the authors and/or other copyright owners and it is a condition of accessing publications that users recognise and abide by the legal requirements associated with these rights.

- Users may download and print one copy of any publication from the public portal for the purpose of private study or research.

- You may not further distribute the material or use it for any profit-making activity or commercial gain

- You may freely distribute the URL identifying the publication in the public portal

If you believe that this document breaches copyright please contact us providing details, and we will remove access to the work immediately and investigate your claim. 


\title{
Hybrid grating reflectors: Origin of ultrabroad stopband
}

\author{
Gyeong Cheol Park, Alireza Taghizadeh, and II-Sug Chung ${ }^{\text {a) }}$ \\ DTU Fotonik, Department of Photonics Engineering, Technical University of Denmark, \\ DK-2800 Kgs. Lyngby, Denmark
}

(Received 4 January 2016; accepted 29 March 2016; published online 7 April 2016)

\begin{abstract}
Hybrid grating (HG) reflectors with a high-refractive-index cap layer added onto a high contrast grating (HCG) provide a high reflectance close to $100 \%$ over a broader wavelength range than HCGs. The combination of a cap layer and a grating layer brings a strong Fabry-Perot (FP) resonance as well as a weak guided mode (GM) resonance. Most of the reflected power results from the FP resonance, while the GM resonance plays a key role in achieving a reflectance close to $100 \%$ as well as broadening the stopband. An HG sample with 7 InGaAlAs quantum wells included in the cap layer has been fabricated by directly wafer-bonding a III-V cap layer onto a Si grating layer. Its reflection property has been characterized. This heterogeneously integrated HG reflector may allow for a hybrid III-V on Si laser to be thermally efficient, which has promising prospects for silicon photonics light sources and high-speed operation. (O 2016 AIP Publishing LLC.

[http://dx.doi.org/10.1063/1.4945737]
\end{abstract}

High contrast gratings (HCGs) have attracted much attention as broadband reflectors, especially for verticalcavity surface-emitting lasers (VCSELs), enabling various laser structures. ${ }^{1-5}$ In addition to the broadband high reflectivity, the reflection/transmission phase of an $\mathrm{HCG}^{6-8}$ as well as its derivative with respect to the in-plane wavevector $^{9-11}$ can be engineered. The control of the derivative of the reflection phase enables it to realize photonic heterostructures in in-plane directions, which is analogous to the electronic quantum wells. This property makes the HCG-based vertical cavities an attractive platform for studying polariton lasers ${ }^{12}$ and parity-time symmetry breaking. ${ }^{11}$

These interesting properties of HCGs originate from the interplay of two waveguide modes propagating along the z-direction. The interplay leading to broadband high reflectivity occurs at periods of near sub-wavelength. ${ }^{1,13,14}$ Thus, it is required that the refractive indices of materials surrounding a grating layer be significantly lower than those of the grating, as shown in Fig. 1(a), so as to make all high order diffractions evanescent. Otherwise, the efficiency of the 0 -th order diffraction, that is, the surface-normal reflectance of our interest, cannot be very high, e.g., $>99.9 \%$. Thus, the high contrast condition has been considered as a requisite for the broadband high reflectivity. ${ }^{1}$

Recently, a grating reflector structure referred to as hybrid grating $(\mathrm{HG})^{15}$ or zero-contrast grating ${ }^{16}$ has been reported, which does not rely on this requisite. As shown in Fig. 1(b), an HG consists of a grating layer and a high-refractive-index layer referred to as the cap layer. As shown in Figs. 1(c) and 1(d), for example, HGs can provide a broader stopband than HCGs. ${ }^{15,16}$ They can be designed as a high-quality factor resonator, as well. ${ }^{17}$ The cap layer introduces Fabry-Perot (FP) resonance via the 0 -th order diffraction, as well as the guidedmode (GM) resonance via the \pm 1 -st order diffractions. The role of the 1-st order diffraction in achieving a high reflectance is qualitatively or phenomenologically discussed. ${ }^{15,18}$ Its

${ }^{a)}$ Electronic mail: ilch@fotonik.dtu.dk role in broadening the stopband has not been rigorously investigated. Furthermore, it has never been discussed whether the engineering of the phase and the derivative of phase are also possible for HG reflectors as in HCGs.

From the application point of view, the HG reflector may have a significant impact on the design of hybrid III-V on Si vertical-cavity lasers based on HCGs. ${ }^{19-23}$ The hybrid vertical-cavity lasers are promising for silicon photonics light sources ${ }^{20,21}$ and ultrahigh-speed operation. ${ }^{23}$ In HCGbased hybrid lasers, achieving efficient heat dissipation is a key challenge for continuous wave (CW) operation, due to the poor heat conduction of the low refractive-index surrounding materials, e.g., air or $\mathrm{SiO}_{2}$. A metallic heat spreader or a channel can be a solution. ${ }^{19,22}$ An HG-based cavity consisting of an HG reflector with a gain material in the cap layer, a very thin dummy cavity, and another reflector can reach the lasing condition, since the field intensity in the cap layer can be comparable to that in the cavity. ${ }^{15}$ In the HGbased laser, the heat can be dissipated directly from the cap layer to the Si layer. For this configuration to be realized, the heterogeneous integration of a III-V cap layer with a gain material onto a Si grating needs to be tested.

In this letter, we numerically investigate the role of 0 -th and 1-st order harmonics in achieving high reflectivity as well as broadening the stopband. This reveals that though most of reflected power comes from the reflections involving the 0-th harmonic, the reflections involving the 1-st harmonic play a key role in achieving the broadband high reflectance. Based on this, it is discussed how the properties of HCG can be inherited to HG reflector, as well as the possible difference between TM and TE polarizations. Furthermore, a heterogeneously integrated hybrid grating with a gain material in the cap layer is fabricated and characterized. This shows the fabrication feasibility of this hybrid approach. For numerical studies, a two-dimensional rigorous coupled wave analysis (RCWA) method was used. ${ }^{24,25}$

First, the transmittance contours of an HCG and an HG reflector are compared to obtain an insight on the origin of 
(a)

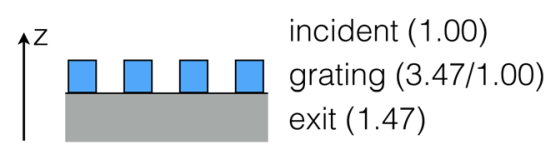

(c)

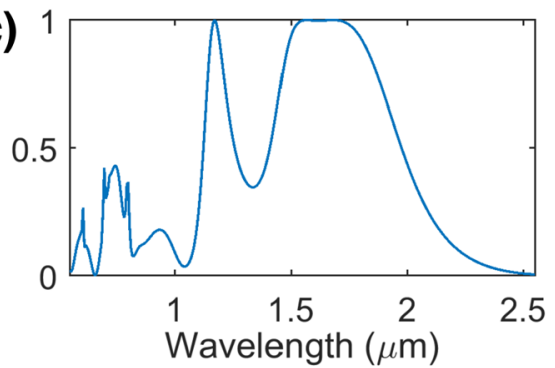

(e)

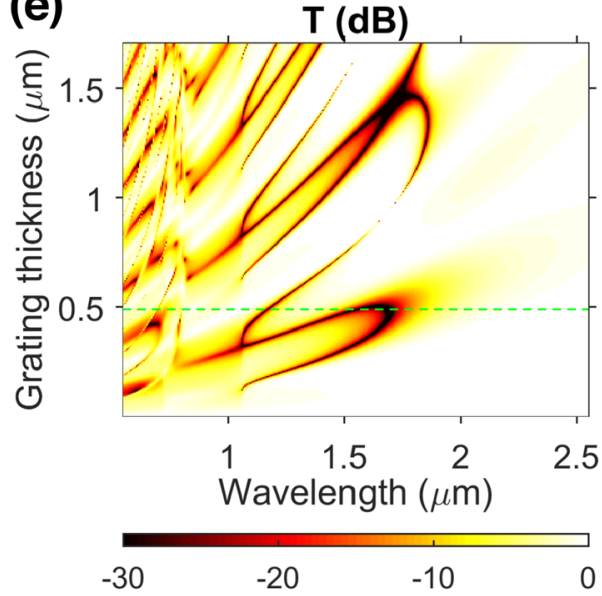

(b)

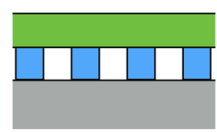

cident (1.00)

cap (3.583)

grating $(3.47 / 1.00)$

exit (1.47)

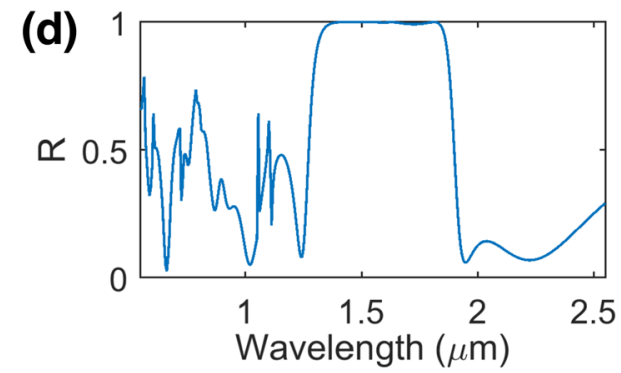

(f)

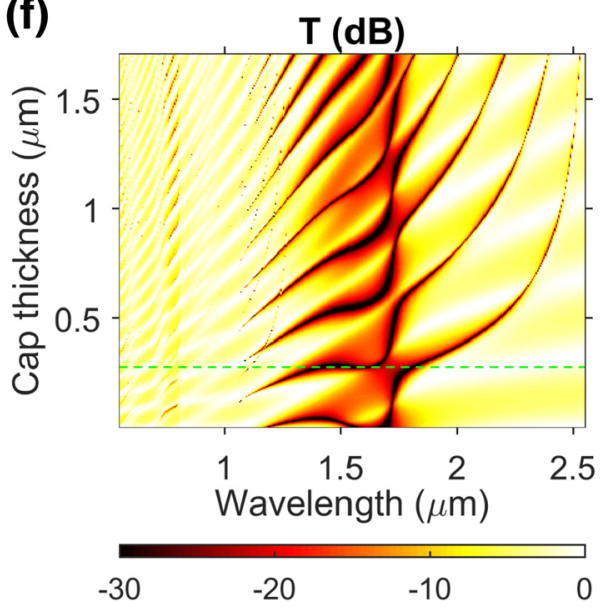

FIG. 1. (a) and (b) Cross-sectional schematics of an HCG and an HG, respectively. Numbers in parentheses are refractive indices. (c) and (d) Reflectance spectra of an HCG and an HG, respectively. The HCG and HG are identical except for the cap layer. The grating period is $720 \mathrm{~nm}$, the grating thickness is $492 \mathrm{~nm}$, and the grating filling factor is $50 \%$. The cap layer thickness is $279 \mathrm{~nm}$. (e) and (f) Transmittance contours of an HCG and an $\mathrm{HG}$ in $\mathrm{dB}$ scale, respectively. For the cases in (e) and (f), other parameters than the grating thickness and cap layer thickness are identical to the cases in (c) and (d), respectively. The green dotted lines in (e) and (f) designate $492 \mathrm{~nm}$ and $279 \mathrm{~nm}$, respectively. differences in HGs from HCGs. The compared HCG and HG reflector are identical except for a cap layer, as described in the caption of Fig. 1. In the contour plot of the HCG shown in Fig. 1(e), the bending of the transmittance dip line occurring around a wavelength of $1.7 \mu \mathrm{m}$ and a grating thickness of $0.5 \mu \mathrm{m}$ provides the broadest stopband of $1.5 \mu \mathrm{m}$ to $1.7 \mu \mathrm{m}$, which is hereafter referred to as "grating stopband." As shown in Fig. 1(f), the narrow transmission contour lines of the HG reflector outside the stopband looks like those of a GM resonance filter, while being distorted within the grating stopband. Due to the distortion, the transmittance dip line becomes very flat around a cap layer thickness of $279 \mathrm{~nm}$, leading to a very broad HG stopband. Since the seemingly distortion of contour lines occurs within the grating stopband, one may guess that the diffraction phase from the grating is abnormal within the grating stopband and strongly influences the phase condition of the GM resonance.

In the cap layer, harmonics of $\{0, \pm 1\}$ and $\{0, \pm 1, \pm 2\}$ are likely to be generated in TM and TE polarizations, respectively. The angle of $m$-th order diffraction, $\theta_{m}$ in the cap layer, is given by $n_{\text {cap }} \sin \left(\theta_{m}\right)=n_{\text {cap }} \sin \left(\theta_{\text {in }}\right)-m \lambda_{0} / \Lambda$, where $n_{\text {cap }}$ is the refractive index of the cap layer, $\lambda_{0}$ is the free-space wavelength, $\theta_{\text {in }}$ is the incident angle, and $\Lambda$ is the grating period. At normal incidence $\left(\theta_{\text {in }}=0\right)$, the diffraction order with a real diffraction angle is limited by $m_{\max }$ $=n_{\text {cap }} \Lambda / \lambda_{0}$. Since the refractive index of the cap layer is typically between 2.9 and 3.6 and the optimal value of $\Lambda / \lambda_{0}$ is about 0.45 and 0.7 for TM and TE polarizations, respectively, ${ }^{14} m_{\max }$ is less than 2.0 for TM polarization and can be larger than 2.0 for TE polarization. Thus, $\{0, \pm 1\}$ are the allowed diffraction orders in the cap layer for TM polarization, while $\{0, \pm 1, \pm 2\}$ are also possible for the TE case. Each diffraction order generates a corresponding harmonic in the cap layer. Here, our discussion is focused on TM polarization and the TE case will be commented on later.

As shown in Figs. 2(a) and 2(b), the possible beam paths contributing to the reflectance of an $\mathrm{HG}$ reflector can be classified into two groups, depending on the involved harmonics in the cap layer. A series of beam paths with the 0-th order harmonic shown in Fig. 2(a) are related to FP resonance, while those with the 1-st order harmonic shown in Fig. 2(b) are related to the GM resonance. The reflection coefficient of a beam path involving the $m$-th harmonic and $i$ roundtrips is represented by $h_{m}(i)$. The summed coefficients, $r_{S}+\sum_{i=1}^{N}$ $h_{0}(i)$ and $r_{S}+\sum_{i=1}^{N} h_{\{0, \pm 1\}}(i)$, are denoted by $s_{0}(N)$ and $s_{\{0, \pm 1\}}(N)$, respectively.

The FP interference via the 0 -th harmonic makes $\left|s_{0}\right|$ high over a broad wavelength range, even outside the grating stopband, as shown in Fig. 3(a). As shown in Fig. 3(b), $\left|r_{A}\right|$ is large, i.e., $>0.8$ over $1.35 \mu \mathrm{m}$ to $1.80 \mu \mathrm{m}$ wavelengths. (a) via 0-th order harmonics

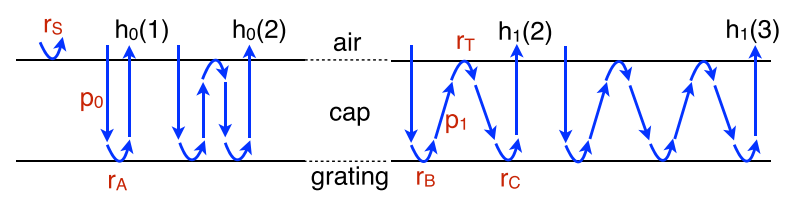

FIG. 2. (a) and (b) Shortest roundtrips in the cap layer via 0-th and 1-st order harmonics, respectively. $r_{i}$ and $p_{j}$ denote interface reflection coefficients and beam propagation factors, respectively. 


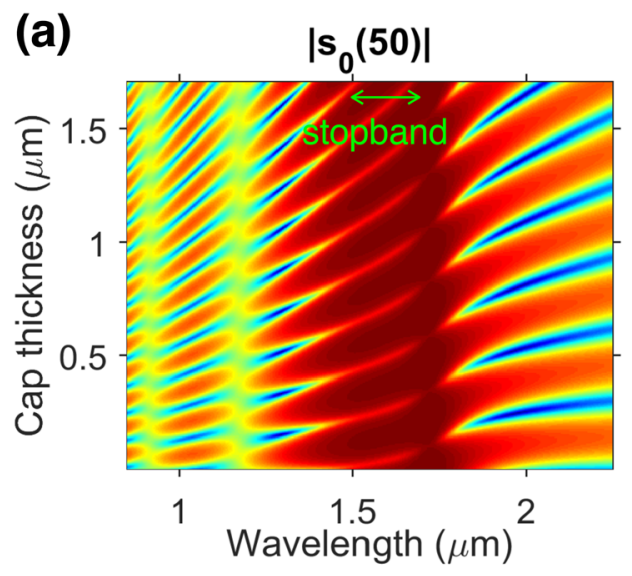

(b)
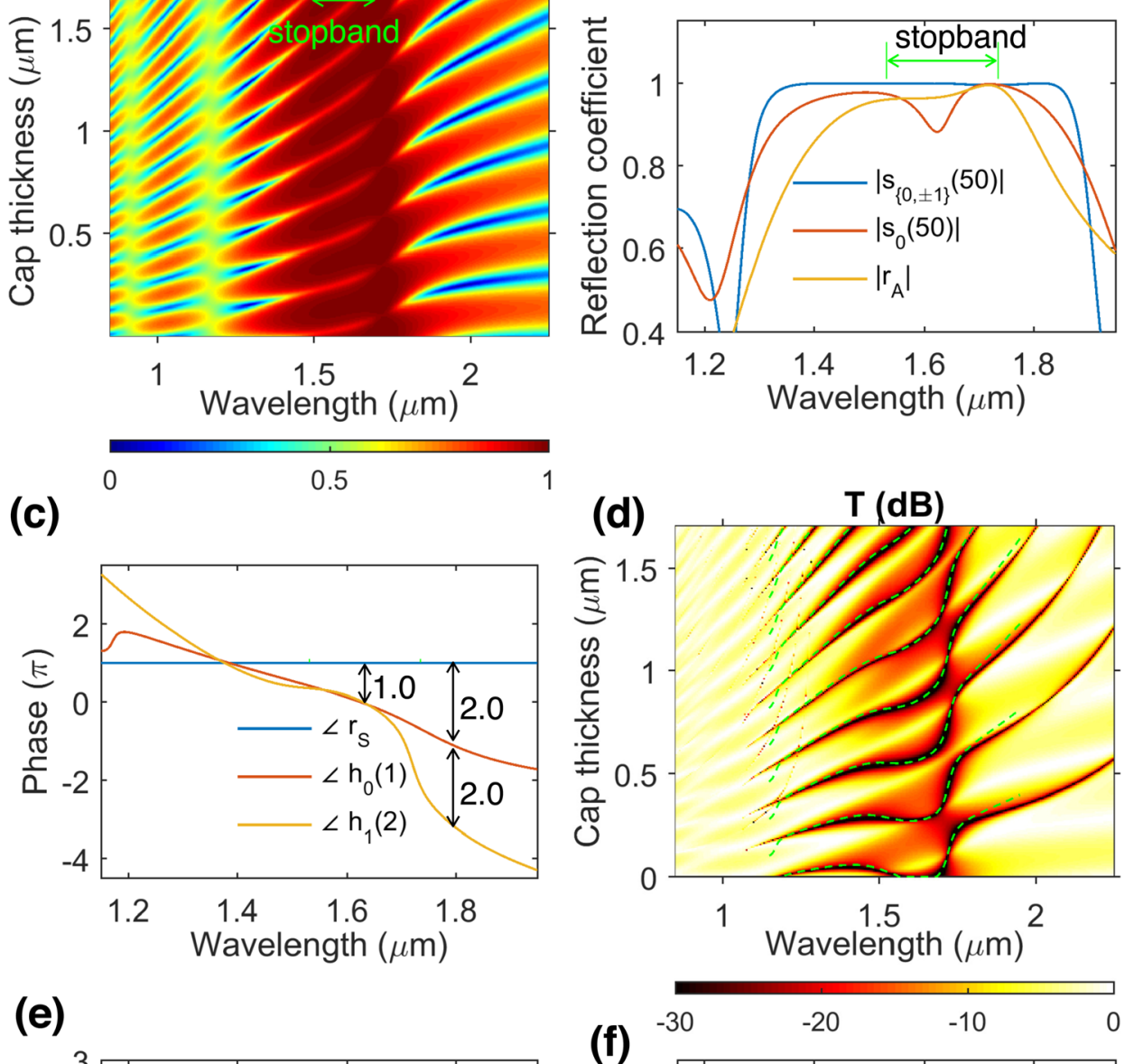

(e)
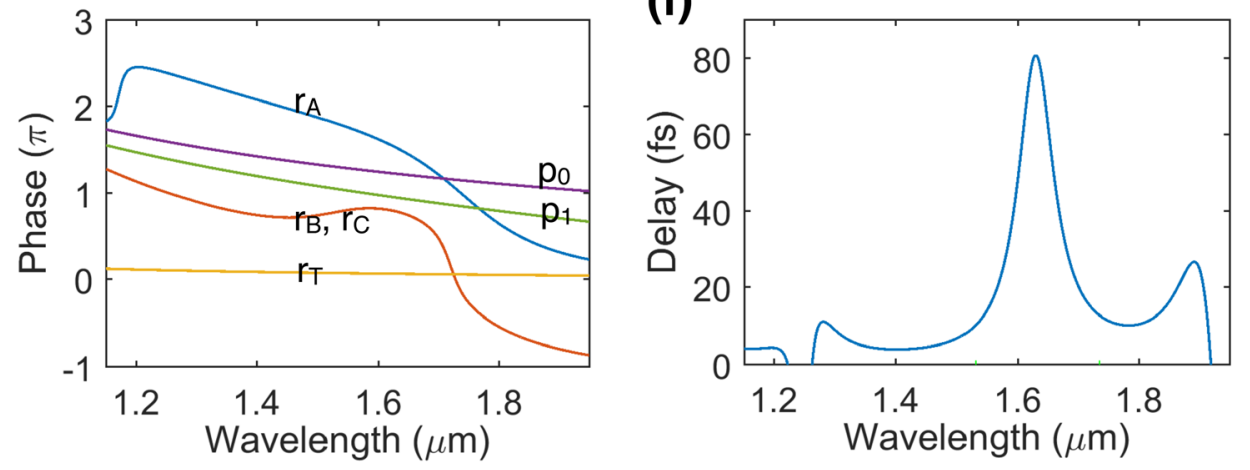

FIG. 3. (a) Contour of reflection coefficient, $\left|s_{0}(50)\right|$ in linear scale. $N=50$ gives saturated values for $s_{0}$ and $s_{\{0, \pm 1\}}$. (b) Reflection coefficient spectra, $r_{A}$, $\left|s_{0}(50)\right|$, and $\left|s_{\{0, \pm 1\}}(50)\right|$. (c) Reflection phase spectra. (d) In-phase condition of $h_{0}(1)$ and $h_{1}(2)$ (green dotted lines) plotted with a transmittance contour in dB scale (identical to Fig. 1(f)). (e) Reflection phase spectra. (f) Reflection delay time of the entire HG structure. For $\left|s_{0}\right|$ and $\left|s_{\{0, \pm 1\}}\right|$ in (b), $\angle h_{0}(1)$ and $\angle h_{1}(2)$ in (c), $\angle p_{0}$ and $\angle p_{1}$ in (e), and in (f), a cap layer thickness of $279 \mathrm{~nm}$ is assumed.
Thus, $\left|s_{0}\right|$ including multiple reflections of $\left|r_{A}\right|$ becomes high at these wavelengths, except around a wavelength of destructive interference. As, for example, shown in Fig. 3(b), the in-phase condition of $r_{S}$ and $h_{0}(1)$ around $1.36-\mu \mathrm{m}$ and 1.80- $\mu$ m wavelengths (see Fig. 3(c)) leads to high $\left|s_{0}\right|$ values, while their out-of-phase condition around $1.63-\mu \mathrm{m}$ results in a local decrease.

Furthermore, the in-phase coupling of the 1-st order harmonic to the 0 -th harmonic increases the reflection coefficient to be close to 1.0. As shown in Fig. 3(c), $h_{1}(2)$ and $h_{0}(1)$ are nearly in-phase over $1.30 \mu \mathrm{m}$ to $1.80 \mu \mathrm{m}$ wavelengths, i.e., $\angle h_{0}(1)-\angle h_{1}(2) \sim 0$ or $2 \pi$. This in-phase coupling makes $\left|s_{\{0, \pm 1\}}\right|$ close to 1.0 , even compensating the local dip around $1.63 \mu \mathrm{m}$ in the $\left|s_{0}\right|$ spectrum. Fig. 3(d) shows that the in-phase condition of $h_{0}(1)$ and $h_{1}(2)$ denoted by green dotted lines matches well with the position of transmittance dip lines within $1.30 \mu \mathrm{m}$ to $1.80 \mu \mathrm{m}$ wavelengths, which supports our explanation above. Outside these wavelengths, the transmittance dip position is determined mainly by the GM resonance condition since $\left|r_{A}\right|$ is no longer high, leading to a weak FP resonance.

In Fig. 3(d), the in-phase condition is significantly distorted within the grating stopband. Thanks to this distortion, the in-phase condition can be met over a broad wavelength range, given that the cap layer thickness is $279 \mathrm{~nm}$. The phase difference, $\angle h_{0}(1)-\angle h_{1}(2)$ is given by $\left(2 \angle p_{0}+\angle r_{A}\right)-\left(2 \angle p_{0}\right.$ $\left.+2 \angle p_{1}+\angle r_{B}+\angle r_{C}+\angle r_{T}\right)$. As shown in Fig. 3(e), both $\angle r_{B}$ and $\angle r_{C}$ have a convex shape within the grating stopband, while other phases monotonically decrease with wavelength. This leads to the distortion of in-phase condition. Since $\angle p_{0}$ and $\angle p_{1}$ increase with the cap layer thickness, one may choose a certain cap layer thickness for obtaining the flattest in-phase condition, which is $279 \mathrm{~nm}$ for the considered case. Figure 3(f) shows a reflection delay spectrum of the $\mathrm{HG},{ }^{26}$ which measures the time taken to reach a steady-state reflectance value when measuring the reflectance in time. The long delay time around 


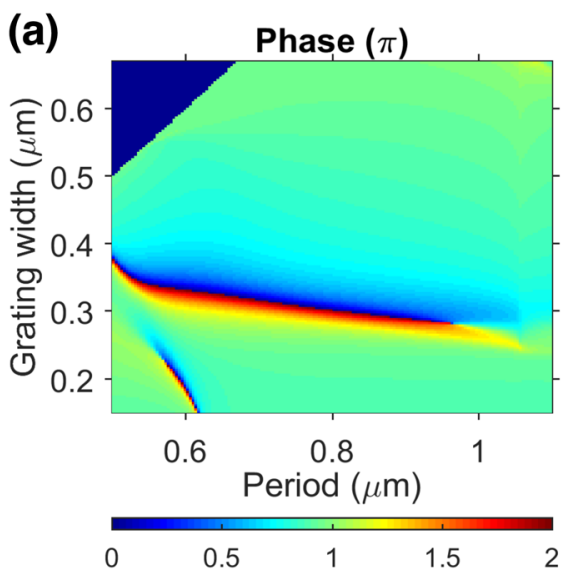

$1.63-\mu \mathrm{m}$ wavelength explains that more number of multiple reflections is needed to compensate the dip in $\left|s_{0}\right|$, as shown in Fig. 3(b).

In the beginning of this letter, we note that the properties of HCGs, i.e., the broadband high reflectance and the feasible control of reflection phase and its derivative, originate from the interplay of two propagating modes. The effect of the two-mode interplay is included in the 0-th order diffraction from an HCG. In HG reflectors, most of reflected power results from the 0 -th order diffraction efficiency, $r_{A}$. Thus, as in $\mathrm{HCGs}^{6}{ }^{6}$ the reflection phase of an $\mathrm{HG}$ can be varied more than $2 \pi$ while maintaining high reflectance values, as shown in Figs. 4(a) and 4(b). Let us shortly discuss the TE polarization case. In order to reach a reflectance close to 1.0 , the beam path related to the 2-nd order harmonic needs to be in phase to those of 0 -th and 1 st order harmonics, which seems to be difficult to be met over a broad wavelength range.

An HG reflector sample was fabricated by heterogeneously integrating a III-V cap layer with an active material onto a Si grating layer, as shown in Fig. 5(a). First, a grating region with a grating period of $720 \mathrm{~nm}$ and a grating width of $360 \mathrm{~nm}$ is formed in the 492-nm-thick Si layer of a silicon on insulator (SOI) wafer, by using electron-beam lithography and dry etching processes. Then, a III-V layer consisting of a 105.5-nm-thick InGaAlAs layer (7 InGaAlAs/InGaAlAs quantum wells with a gain peak at $1.54-\mu \mathrm{m}$ wavelength) and a 191.7-nm thick InP layer is directly wafer-bonded onto the SOI wafer. ${ }^{27,28}$ The direct wafer bonding does not require an expensive alignment process and provides a high yield for wafer-scale as well as die-level bondings. Before the bonding, both III-V and SOI samples are prepared with a RCA-1 process and a plasma treatment, and are pressed at $300{ }^{\circ} \mathrm{C}$ after the bonding by using a bonding machine (EVG 520 $\mathrm{HE})$. Then, the InP substrate is removed by using a wet etching process.

The reflection spectrum of the fabricated sample was characterized by using a free-space setup which consists of a super-continuum laser (SuperK Extreme, NKT Photonics), a polarization filter, and an optical spectrum analyzer. Parabolic gold mirrors are used to guide the light and focus the light at the surface of the $\mathrm{HG}$ reflector sample. In this setup, the incident angle of light onto the sample is $\sim 3.5^{\circ}$. The reflection spectrum of a gold mirror measured by using the same setup is used for calibration. As shown in Figs. 5(b) and 5(c), the calculated and measured spectra with a TM-polarized incident light shows a good agreement. The absorption in quantum wells can decrease the reflectance by up to $2 \%$, depending on wavelengths. The overall blue shift of the calculated spectrum can be attributed to the refractive index value that we assume for InAlGaAs layer as well as the neglected dispersion of refractive indices. The reflectance of HGs is tolerable against typical fabrication deviations. ${ }^{15}$

In summary, the cap layer introduces FP resonance as well as GM resonance. The in-phase condition of FP and GM resonances leads to a very high reflectance close to $100 \%$. Though most of the reflected power comes from the FP resonance, the GM resonance plays a key role in broadening the

(a)

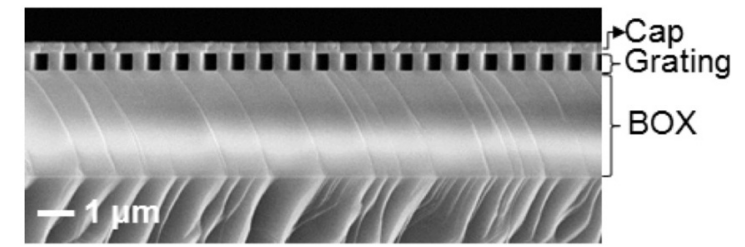

(b)

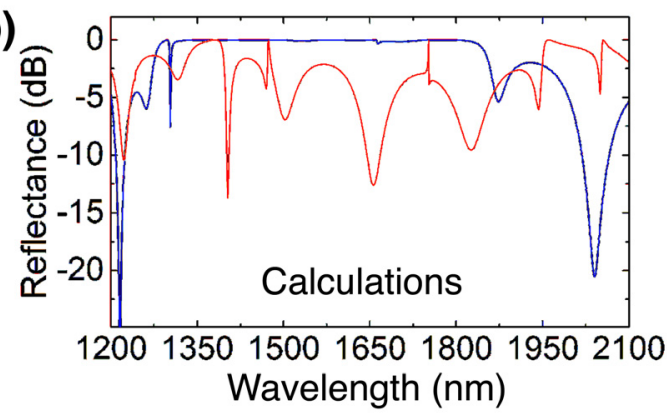

(c)

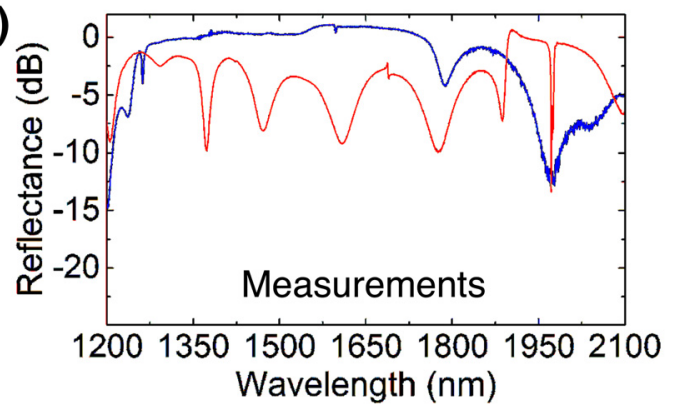

FIG. 5. (a) A scanning electron microscope (SEM) image of a fabricated HG reflector sample. (b) and (c) Calculated and measured reflectance spectra at an incidence angle of $3.5^{\circ}$, respectively (blue: TM polarization and red: TE polarization). 
stopband. The fabrication and characterization of a heterogeneously integrated HG sample shows the fabrication feasibility of an HG-based Si-integrated laser, which has promising prospects for silicon photonics light sources and high-speed laser applications.

The authors gratefully acknowledge the support from the Danish Council for Independent Research through FTP project (0602-01885B) as well as the Innovation Fund Denmark through the HOT project (5106-00013B).

${ }^{1}$ C. F. R. Mateus, M. C. Y. Huang, L. Chen, C. J. Chang-Hasnain, and Y. Suzuki, IEEE Photonics Technol. Lett. 16, 1676 (2004).

${ }^{2}$ M. C. Y. Huang, Y. Zhou, and C. J. Chang-Hasnain, Nat. Photonics 1, 119 (2007).

${ }^{3}$ M. C. Y. Huang, Y. Zhou, and C. J. Chang-Hasnain, Nat. Photonics 2, 180 (2008).

${ }^{4}$ P. Gilet, N. Olivier, P. Grosse, K. Gilbert, A. Chelnokov, I.-S. Chung, and J. Mørk, Proc. SPIE 7615, 76150J (2010).

${ }^{5}$ T.-T. Wu, S.-H. Wu, T.-C. Lu, and S.-C. Wang, Appl. Phys. Lett. 102, 081111 (2013).

${ }^{6}$ D. Fattal, J. Li, Z. Peng, M. Fiorentino, and R. G. Beausoleil, Nat. Photonics 4, 466 (2010).

${ }^{7}$ F. Lu, F. G. Sedgwick, V. Karagodsky, C. Chase, and C. J. ChangHasnain, Opt. Express 18, 12606 (2010).

${ }^{8}$ L. Carletti, R. Malureanu, J. Mørk, and I.-S. Chung, Opt. Express 19, 23567 (2011).

${ }^{9}$ C. Sciancalepore, B. B. Bakir, X. Letartre, J.-M. Fedeli, N. Olivier, D. Bordel, C. Seassal, P. Rojo-Romeo, P. Regreny, and P. Viktorovitch, J. Lightwave Technol. 29, 2015 (2011).
${ }^{10}$ Z. Wang, B. Zhang, and H. Deng, Phys. Rev. Lett. 114, 073601 (2015).

${ }^{11}$ A. Taghizadeh, J. Mørk, and I.-S. Chung, Appl. Phys. Lett. 107, 181107 (2015).

${ }^{12}$ B. Zhang, S. Brodbeck, Z. Wang, M. Kamp, C. Schneider, S. Höfling, and H. Deng, Appl. Phys. Lett. 106, 051104 (2015).

${ }^{13}$ V. Karagodsky and C. J. Chang-Hasnain, Opt. Express 20, 10888 (2012).

${ }^{14}$ I.-S. Chung, Opt. Express 23, 16730 (2015).

${ }^{15}$ A. Taghizadeh, G. C. Park, J. Mørk, and I.-S. Chung, Opt. Express 22, 21175 (2014).

${ }^{16}$ R. Magnusson, Opt. Lett. 39, 4337 (2014).

${ }^{17}$ A. Taghizadeh, J. Mørk, and I.-S. Chung, Opt. Express 23, 14913 (2015).

${ }^{18}$ H. Tian, X. Cui, Y. Du, P. Tan, G. Shi, and Z. Zhou, Opt. Express 23, 27174 (2015).

${ }^{19}$ I.-S. Chung and J. Mørk, Appl. Phys. Lett. 97, 151113 (2010).

${ }^{20}$ C. Sciancalepore, B. B. Bakir, X. Letartre, J. Harduin, N. Olivier, C. Seassal, J.-M. Fedeli, and P. Viktorovitch, IEEE Photonics Technol. Lett. 24, 455 (2012).

${ }^{21}$ G. C. Park, W. Xue, A. Taghizadeh, E. Semenova, K. Yvind, J. Mørk, and I.-S. Chung, Laser Photonics Rev. 9, L11 (2015).

${ }^{22}$ J. Ferrara, W. Yang, L. Zhu, P. Qiao, and C. J. Chang-Hasnain, Opt. Express 23, 2512 (2015).

${ }^{23}$ G. C. Park, W. Xue, J. Mørk, E. Semenova, and I.-S. Chung, Integrated Photonics Research, Silicon and Nanophotonics JT5A.2 (2015).

${ }^{24}$ M. G. Moharam, E. B. Grann, D. A. Pommet, and T. K. Gaylord, J. Opt. Soc. Am. A 12, 1068 (1995).

${ }^{25}$ L. Li, J. Opt. Soc. Am. A 13, 1870 (1996).

${ }^{26}$ I.-S. Chung, V. Iakovlev, A. Sirbu, A. Mereuta, A. Caliman, E. Kapon, and J. Mørk, IEEE J. Quantum Electron. 46, 1245-1253 (2010).

${ }^{27}$ D. Liang and J. E. Bowers, J. Vac. Sci. Technol. B 26, 1560 (2008).

${ }^{28}$ M. J. R. Heck, J. F. Bauters, M. L. Davenport, J. K. Doylend, S. Jain, G. Kurczveil, S. Srinivasan, Y. Tang, and J. E. Bowers, IEEE J. Sel. Top. Quantum Electron. 19, 6100117 (2013). 